\title{
Peruvian Agricultural Research
}

\author{
ISSNe 2706-9397
}

Homepage: http://revistas.unjfsc.edu.pe/index.php/PeruvianAgriculturalResearch CUniversidad Nacional José Faustino Sánchez Carrión, Lima, Perú

Recibido: Noviembre 23, 2021 / Aceptado: Diciembre 30, 2021

3(2), 52-56, 2021

\section{Evaluación del comportamiento agronómico de dlos cultivares de papa en dos densidades de siembra en Barranca, Lima}

\author{
Evaluation of the agronomic performance of two potato cultivars and two planting \\ densities in Barranca, Lima
}

\section{P. $\operatorname{Vargas}^{1 *}\left(\mathbb{D}\right.$, D. A. Alarcón ${ }^{2} \mathbb{D}$, A. A. Espinoza $^{2} \mathbb{D}$}

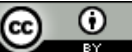

https://doi.org/10.51431/par.v3i2.701

\section{Resumen}

Objetivos: Evaluar el comportamiento agronómico de dos cultivares comerciales de papa (Solanum tuberosum) bajo dos densidades de siembra en la localidad de Vinto, Barranca. Metodología: La investigación se desarrolló en condiciones de Costa en la localidad de Vinto, distrito de Barranca (Lima) a una altitud de $227 \mathrm{~m} \mathrm{s.} \mathrm{n.} \mathrm{m.}$ Se utilizó el diseño de bloques completos al azar con arreglo factorial $2 \times 2$. El primer factor correspondió a los cultivares Única y Bicentenaria y el segundo factor, a las densidades de siembra de 3,33 y 4,00 tubérculossemilla por $\mathrm{m}^{2}$, con cuatro réplicas por tratamiento. Las características evaluadas fueron peso de follaje, peso de tubérculos por planta, número de tubérculos por planta, peso de tubérculos y rendimiento. Para la comparación de medias de tratamiento, se utilizó la prueba de Tukey al 5\% de significación. Resultados: Se halló interacción significativa entre los cultivares y las densidades de siembra en peso fresco de follaje y de tubérculos por planta. El cultivar Bicentenaria fue superior al cv. Única al producir significativamente mayor peso de follaje en ambas densidades de siembra, sin embargo para peso de tubérculos por planta, Única fue superior a Bicentenaria. Con respecto a número de tubérculos por planta, Bicentenaria fue superior a Única, en tanto que para rendimiento de tubérculos, los mayores valores fueron obtenidos por el cultivar Única. Conclusiones: Se concluye que bajo las condiciones de Barranca el cv. Única fue superior en rendimiento de tubérculos al cv. Bicentenaria y que a una mayor densidad de siembra, se obtuvo un incremento aproximado de $17 \%$ en el rendimiento total por hectárea.

Palabras clave: Densidad de siembra, evaluación agronómica, mejoramiento genético, Solanum tuberosum, rendimiento

\begin{abstract}
Objectives: To evaluate the performance of two commercial potato cultivars (Solanum tuberosum) under two planting densities in Vinto, Barranca. Methodology: The research was developed under coastal conditions in Vinto, district of Barranca (Lima) at 227 masl. A randomized complete block design was used with a $2 \times 2$ factorial arrangement. The first factor corresponded to Unica and Bicentenaria cultivars and the second factor to the planting densities of 3.33 and 4.00 seed tubers per $\mathrm{m}^{2}$, with four replications per treatment. The characteristics evaluated were foliage weight, tuber weight per plant, number of tubers per plant, and tuber yield. For the comparison of treatment means, the Tukey test was used at $5 \%$ significance. Results: It has been observed a significant interaction between cultivars and planting densities in fresh weight of foliage and tuber weight per plant. The cultivar Bicentenaria was superior to Unica by producing a significantly higher weight of foliage in both planting densities, however, for the weight of tubers per plant, Unica was superior to Bicentenaria. Regarding the number of tubers per plant, Bicentenaria was superior to Unica, while for tuber yield, the highest values were obtained by cv. Unica cultivar. Conclusions: It is concluded that under the conditions of Vinto (Barranca) cv. Unica was superior in tuber yield to $\mathrm{cv}$. Bicentenaria and that at a higher planting density, an increase of $17 \%$ in the total yield per hectare was obtained.
\end{abstract}

Keywords: Planting density, agronomic evaluation, plant breeding, Solanum tuberosum, yield

\footnotetext{
${ }^{1}$ Departamento Académico de Ingeniería, Universidad Nacional de Barranca, Lima, Perú.

${ }^{2}$ Escuela de Agronomía, Universidad Nacional de Barranca, Lima, Perú.

*Autor para correspondencia: lvargas@unab.edu.pe

52
} 


\section{Introducción}

La papa (Solanum tuberosum L.), a nivel mundial es uno de los cuatro alimentos más consumidos, después del trigo, arroz y maíz (Seminario-Cunya et al., 2018) y en el Perú, es el tercer cultivo de importancia económica, después de arroz y café. Así, para la campaña 2019-2020 se sembraron 338 mil hectáreas de papa con un rendimiento promedio de $15,65 \mathrm{t}$ $\mathrm{ha}^{-1}$, no obstante, los mayores rendimientos se obtuvieron en los departamentos de Arequipa $\left(35,00 \mathrm{t} \mathrm{ha}^{-1}\right)$, Ica $\left(34,99 \mathrm{t} \mathrm{ha}^{-1}\right)$ y Lima $(21,21 \mathrm{t}$ ha $^{-1}$ ) (Ministerio de Desarrollo Agrario y Riego, 2021); su cultivo está cargo principalmente de pequeños agricultores (Cervantes y Quevedo, 2020).

Uno de los grandes problemas que se viene observando en este cultivo es que las diversas variedades cultivadas no presentan características adecuadas para atender la demanda creciente de las comidas rápidas, razón por lo cual las empresas involucradas en ese rubro tienen que importar productos procesados de papa (Amaya, 2020); en el año 2020 se importaron 19,47 mil toneladas por un valor de 16,81 millones de dólares, siendo los Países Bajos el principal proveedor con el 74,4 \% (Superintendencia Nacional de Administración Tributaria, 2021).

En ese contexto, la necesidad de desarrollar variedades que puedan atender esa demanda ya se viene ejecutando, por lo que actualmente en el mercado se puede disponer de la variedad Única, que se cultiva tanto a nivel nacional como internacional, y que destaca por sus atributos de calidad para fritura, altos rendimientos y fácil adaptabilidad a diversos climas en el mundo (Centro Internacional de la Papa, 2018). Esta variedad se caracteriza por presentar tubérculos oblongos con ojos superficiales y que puede mostrar ciertas protuberancias cuando se presentan problemas ambientales o de manejo del cultivo (Gutiérrez et al., 2007).

En esa misma línea, la Universidad Nacional José Faustino Sánchez Carrión en cooperación con el Centro Internacional de la Papa y las Universidades de Barranca y de Cañete han venido desarrollando un nuevo material genético (Agroperu, 2021) con características adecuadas para el procesamiento, que se ha denominado cv. Bicentenaria (clon UH-24) en el Registro Nacional de Cultivares Comerciales del Servicio Nacional de Sanidad Agraria, con muy buena capacidad de adaptación a diferentes ambientes, que se caracteriza por su abundante follaje de color verde intenso, con tubérculos uniformes y ojos superficiales, de piel crema y pulpa blanca, con capacidad de producir 8 tubérculos por planta, y obtener rendimientos promedios de 44,8 t ha ${ }^{-1}$; así también, el tubérculo se caracteriza por presentar $18,4 \%$ de sólidos solubles, con color adecuado y reducido defecto en la producción de hojuelas, y calidad óptima en tiras para pollerías (Contreras-Liza, 2020).

En ese contexto, la presente investigación tuvo como propósito evaluar el comportamiento agronómico de los cultivares comerciales de papa para procesamiento, Única y Bicentenaria bajo dos densidades de siembra en la localidad de Vinto, Barranca.

\section{Metodología}

El experimento de campo conducido durante los meses de junio a diciembre del 2020 en la propiedad de un pequeño agricultor, localizado en Vinto, distrito de Barranca en las coordenadas geográficas $10^{\circ} 41^{\prime} 11^{\prime \prime}$ latitud sur, $77^{\circ} 41^{\prime} 13$ " longitud oeste y a una altitud de $227 \mathrm{~m} \mathrm{~s}$. n. m. El clima de la región, según la clasificación de Köppen es del tipo BWh, que se caracteriza por su ambiente árido y seco con temperatura media anual de $18,6^{\circ} \mathrm{C}$ y precipitación anual menor a $19 \mathrm{~mm}$. El suelo del área utilizada corresponde a la textura franco arenoso.

Se utilizó un arreglo factorial de 2 x 2 bajo el diseño de bloques completos al azar. Los factores en estudio fueron: a) cultivares (Bicentenaria y Única) y b) densidades de siembra $(3,33$ y 4,00 tubérculos-semilla por $\mathrm{m}^{2}$ ), con cuatro repeticiones por tratamiento. E1 tamaño de la unidad experimental fue de $20 \mathrm{~m}^{2}$. La conducción del experimento correspondió al manejo comercial, desde la siembra hasta la cosecha. Para la fertilización del cultivo, se aplicó 250-210-200 kg ha-1 de N-P $\mathrm{O}_{5}-\mathrm{K}_{2} \mathrm{O}$ y $30 \mathrm{t} \mathrm{ha}^{-1}$ de guano de pollo. La principal plaga que afectó al cultivo fue la mosca minadora (Liriomyza huidobrensis) por lo que se realizaron 
las aplicaciones necesarias de insecticidas para su control. Con respecto a las enfermedades foliares, estas no fueron significativas durante la campaña.

Las variables evaluadas fueron peso de follaje, peso de tubérculos por planta, número de tubérculos por planta, peso de tubérculo y rendimiento. Para ello se procedió al muestreo de los dos surcos centrales, por cada unidad experimental. Los datos obtenidos, previa prueba de normalidad y homogeneidad de varianzas, fueron sometidos al análisis de varianza $(P \leq$ 0,05). Para la comparación de medias se utilizó la prueba estadística de Tukey al 5\% de probabilidad. Los datos obtenidos fueron procesados con el software SAS/STAT, versión SAS ${ }^{\circledR}$.

\section{Resultados y discusión}

En la Tabla 1 se presentan los resultados del análisis de varianza para las variables evaluadas. Se observa que existe interacción entre cultivares y densidades de siembra para peso de follaje y peso de tubérculos por planta. Para número de tubérculos por planta y peso promedio por tubérculo solo se ha mostrado diferencias significativas entre los genotipos en estudio. En el caso del rendimiento, las diferencias significativas son tanto entre genotipos como entre la densidad de siembra. Los coeficientes de variabilidad se encuentran dentro del rango aceptable, tal como lo refiere Pimentel-Gomes (2009).

En la Tabla 2, con respecto al peso del follaje, se aprecia que el cv. Bicentenaria mostró superioridad sobre el cv. Única para ambas densidades; sin embargo, obtuvo menor peso de tubérculos por planta, tal como se aprecia en la Tabla 3. En efecto, el hecho que la planta presente mayor biomasa en el follaje no garantiza una mayor producción, pues puede estar ocurriendo que gran parte de los fotosintatos se estén utilizando en la formación de estructuras vegetativas y retrasando el inicio de la tuberización; mientras que una tuberización temprana puede dar lugar a la formación de menor follaje, tal como lo refiere Jerez y Martín (2012). Asimismo, Silva et al. (2018), señalan que el excesivo desarrollo foliar puede ocasionar fallas en la partición de los asimilados provocando la reducción del rendimiento.

Con respecto al número de tubérculos por planta (Tabla 4), el cv. Bicentenaria mostró mayor prolificidad, pero menor peso promedio por tubérculo en comparación al cv. Única. Este hecho es explicable porque a mayor número de tubérculos implica menor recepción de los fotoasimilados, tal como lo refiere Jerez et al.

Tabla 1

Resumen de análisis de varianza para peso de follaje (PF), peso de tubérculos por planta (PTPP), número de tubérculos por planta (NTC), peso de tubérculos (PT) y rendimiento (R)

\begin{tabular}{|c|c|c|c|c|c|c|}
\hline \multirow{2}{*}{$\begin{array}{l}\text { Fuentes de } \\
\text { variabilidad }\end{array}$} & \multirow{2}{*}{$\begin{array}{c}\text { Grados de } \\
\text { libertad }\end{array}$} & \multicolumn{5}{|c|}{ Cuadrados medios } \\
\hline & & $\mathrm{PF}(\mathrm{g})$ & PTPP (g) & NTC & $\mathrm{PT}(\mathrm{g})$ & $\mathrm{R}\left(\mathrm{t} \mathrm{ha}^{-1}\right)$ \\
\hline Bloques & 2 & $5547,20 * *$ & $20529,19 \mathrm{~ns}$ & $2,72 \mathrm{~ns}$ & $434,78 \mathrm{~ns}$ & $21,43 \mathrm{~ns}$ \\
\hline Cultivares (C) & 1 & $301321,20 * *$ & $938600,49 * *$ & $23,52 * *$ & $31444,99 * *$ & $964,42 * *$ \\
\hline Densidades (D) & 1 & $386,24 \mathrm{~ns}$ & $8537,07 \mathrm{~ns}$ & $0,15 \mathrm{~ns}$ & $3,04 \mathrm{~ns}$ & $336,85 * *$ \\
\hline$C^{*} \mathrm{D}$ & 1 & $3173,30 *$ & $93197,48 *$ & $1,92 \mathrm{~ns}$ & $23,13 \mathrm{~ns}$ & $51,38 \mathrm{~ns}$ \\
\hline Error & 6 & 272,32 & 11279,17 & 0,9 & 617,81 & 13,68 \\
\hline Total & 11 & & & & & \\
\hline CV (\%) & & 4,87 & 5,16 & 8,68 & 12,69 & 5,45 \\
\hline Promedio & & 338,87 & 2059,44 & 10,96 & 195,89 & 67,88 \\
\hline
\end{tabular}

ns: no significativo; *: significativo al 5\%; **: significativo al $1 \%, \mathrm{CV}$, coeficiente de variabilidad (\%). 
Tabla 2

Interacción entre cultivar y densidad de siembra para peso de follaje ( $g$ )

\begin{tabular}{lcc}
\hline \multirow{2}{*}{ Cultivar } & \multicolumn{2}{c}{ Densidad (tubérculos-semillas $\mathrm{m}^{-2}$ ) } \\
\cline { 2 - 3 } & 3,33 & 4,00 \\
\hline Bicentenaria & $475,40^{\mathrm{Aa}}$ & $519,27^{\mathrm{Aa}}$ \\
Única & $191,00^{\mathrm{Ba}}$ & $169,82^{\mathrm{Ba}}$ \\
\hline
\end{tabular}

Medias con una letra mayúscula en común en la columna, y con una letra minúscula en común en la fila no son significativamente diferentes $(P>0,05)$.

Tabla 3

Interacción entre cultivar y densidad para peso de tubérculos por planta $(g)$

\begin{tabular}{lcc}
\hline \multirow{2}{*}{ Cultivar } & \multicolumn{2}{c}{ Densidad (tubérculos-semillas m ${ }^{-2}$ ) } \\
\cline { 2 - 3 } & 24,33 & 4,00 \\
\hline Única & $2453,91^{\mathrm{Aa}}$ & $224,31^{\mathrm{Aa}}$ \\
Bicentenaria & $1718,31^{\mathrm{Ba}}$ & $1841,22^{\mathrm{Ba}}$ \\
\hline A,B,a Medias con una letra mayúscula en común en la columna, \\
\multicolumn{2}{l}{ y con una letra minúscula en común en la fila no son } \\
significativamente diferentes $(P>0,05)$.
\end{tabular}

Tabla 4

Número de tubérculos por planta (NTC) y peso del tubérculo (PT), según genotipo

\begin{tabular}{lcc}
\hline Cultivar & NTC & PT (g) \\
\hline Bicentenaria & $12,36^{\mathrm{a}}$ & $144,70^{\mathrm{b}}$ \\
Unica & $9,56^{\mathrm{b}}$ & $247,08^{\mathrm{a}}$ \\
\hline
\end{tabular}

${ }^{\mathrm{a}, \mathrm{b}}$ Medias con una letra común no son significativamente diferentes $(P>0,05)$.

(2017) quienes señalan que la formación de los tubérculos y el respectivo llenado, dependen de la disponibilidad de fotoasimilados y de la habilidad de éstos para acumularlos.

Para rendimiento, se aprecia que el cultivar Única superó significativamente al cv. Bicentenaria (Tabla 5). Este resultado puede explicarse porque la respuesta de los genotipos en los diferentes ambientes y épocas de siembra es variable, tal como lo evidencian las investigaciones desarrolladas por ContrerasLiza (2020) y Gutiérrez et al. (2007). Con respecto a la densidad de siembra (Tabla 6), el mayor rendimiento de tubérculos por hectárea le correspondió a 4,00 plantas por $\mathrm{m}^{2}$ con un
Tabla 5

Comparación de rendimiento total de tubérculos entre cultivares de papa

\begin{tabular}{lc}
\hline Genotipo & Rendimiento $\left(\mathrm{t} \mathrm{ha}^{-1}\right)$ \\
\hline Única & $144,70^{\mathrm{b}}$ \\
Bicentenaria & $76,85^{\mathrm{a}}$ \\
\hline a,b Medias con una letra común no son significativamente
\end{tabular}
diferentes $(P>0,05)$.

Tabla 6

Comparación del rendimiento de tubérculos entre densidades de siembra

\begin{tabular}{lc}
\hline $\begin{array}{l}\text { Densidad } \\
\left(\text { tubérculos semillas } \mathrm{m}^{-2}\right)\end{array}$ & $\begin{array}{c}\text { Rendimiento } \\
\left(\mathrm{t} \mathrm{ha}^{-1}\right)\end{array}$ \\
\hline 4,00 & $73,18^{\mathrm{a}}$ \\
3,33 & $62,58^{\mathrm{b}}$ \\
\hline a,b Medias con una letra común no son significativamente \\
\multicolumn{2}{c}{ diferentes $(P>0,05)}$.
\end{tabular}

incremento aproximado de $17 \%$ en este carácter respecto a la menor densidad; esto es explicable porque al aumentar el número de plantas por unidad de área, los rendimientos tienden a incrementarse, tal como lo refieren Chehaibi et al. (2013) y Silva et al. (2018), quienes manifiestan que el aumento de la densidad de siembra promueve la formación de mayor área foliar, y por ende una mayor captación de la radiación fotosintéticamente activa, incidiendo directamente en el aumento del rendimiento.

Por otra parte, Almeida et al. (2016) mencionan que el rendimiento de la papa depende de su crecimiento vegetativo y de la duración del periodo de crecimiento de los tubérculos, es decir, mientras se mantenga verde por más tiempo, mayor será el aprovechamiento de los fotoasimilados por los tubérculos en crecimiento, tal como fue observado en el cultivar Única.

\section{Conclusiones}

Se concluye que bajo las condiciones de Barranca (Lima) el cv. Única fue superior en rendimiento al cv. Bicentenaria y que la mayor densidad de siembra favoreció un incremento de aproximadamente $17 \%$ en el rendimiento de tubérculos en ambos cultivares de papa. 


\section{Referencias}

Agroperu. (2021). Clon UH-24, la papa del Bicentenario. https://www.agroperu.pe/clonuh-24-la-papa-del-bicentenario/

Almeida, F., Sánchez, J. A., Torres, W., \& Cabrera, J. A. (2016). Efectos de diferentes distancias de plantación y calibres de tubérculossemilla sobre algunas características morfo-productivas de la papa en Huambo, Angola. Cultivos tropicales, 37(2), 88-95. http://scielo.sld.cu/scielo.php?script=sci arttext\&pid=S0258-59362016000200010

Amaya, J. (2020). Papa procesada importada. https://gestion.pe/economia/minagri-el-10-dela-papa-procesada-que-se-consume-en-el-paises-importada-noticia/?ref=gesr

Centro Internacional de la Papa. (2018). UNICA, una papa adaptable $y$ productiva para los agricultores de todo el mundo. https:// cipotato.org/es/blog-es/unica-papa-adaptableproductiva-agricultores-mundo/

Cervantes, D. M., \& Quevedo, J. M. (2020). The industrialization of potatoes and the socioeconomic development of family farming in Lima, Peru. Peruvian Agricultural Research, 2 (2), 41-47. https://doi.org/10.51431/par. v2i2.640

Chehaibi, S., Douh, B., Mohandes, E. B., \& Hadj, M. W. (2013). Impact of mechanical planting depth and density on agronomic parameters of organic potato Solanum tuberosum L. Journal of Applied Horticulture, 15(2), 147-149. https:// doi.org/10.37855/jah.2013.v15i02.29

Contreras-Liza, S. E. (2020). Bicentenaria, nueva variedad de papa en la región Lima. https:// www.researchgate.net/publication/343021163 BICENTENARIA_NUEVA_VARIEDAD_ DE_PAPA_EN_REGION_LIMA
Gutiérrez, R., Espinoza, J., \& Bonierbale, M. (2007). ÚNICA: variedad peruana para mercado fresco y papa frita con tolerancia y resistencia para condiciones climáticas adversas. Revista Latinoamericana de la Papa, 14(1), 41-50. http://papaslatinas.org/index.php/rev-alap/ article/view/143

Jerez, E., Martín, R., \& Morales, D. (2017). Evaluación del crecimiento y composición por tamaño de tubérculos de plantas de papa para semilla. Cultivos tropicales, 38(4), 102-110. http://scielo.sld.cu/pdf/ctr/v38n4/ctr15417.pdf

Jerez, E., \& Martín, R. (2012). Comportamiento del crecimiento y el rendimiento de la variedad de papa (Solanum tuberosum L.) Spunta. Cultivos tropicales, 33(4), 53-58. http://scielo.sld.cu/pdf/ ctr/v33n4/ctr07412.pdf

Ministerio de Agricultura y Riego. (2021). Series históricas. https://1bestlinks.net/FgMbC

Pimentel-Gomes, F. (2009) Curso de estadistica experimental (15.a ed.). FEALQ (Fundação de Estudos Agrários Luiz de Queiroz).

Seminario-Cunya, J. F., Villanueva-Guevara, R., \& Valdez-Yopla, M. H. (2018). Rendimiento de cultivares de papa (Solanum tuberosum L.) amarillos del grupo Phureja. Agronomia Mesoamericana, 29(3), 638-653. https://www.redalyc.org/ journal/437/43756297012/43756297012.pdf

Silva, A., Albornoz, C., \& Criollo, H. (2018). Efecto del potasio y la densidad de siembra en la producción de papa Solanum tuberosum Grupo Phureja var. Criolla Guaneña. Temas Agrarios, 23(1), 37-46. https://doi.org/10.21897/rta. v23i1.1145

Superintendencia Nacional de Administración Tributaria. (2021). Papa procesada: Partida arancelaria 2004100000. http://www. aduanet.gob.pe/cl-ad-itestadispartida/ resumenPPaisS01Alias 